\title{
The status of medical expulsive therapy in the age of evidence-based medicine
}

\author{
Julian Veser, Christian Seitz \\ Department of Urology, Medical University Vienna, Vienna, Austria \\ Correspondence to: Christian Seitz. Department of Urology, Medical University Vienna, Vienna, Austria. Email: drseitz@gmx.at. \\ Provenance: This is an invited Editorial commissioned by Section Editor Peng Zhang (Department of Urology, Zhongnan Hospital of Wuhan \\ University, Wuhan, China). \\ Comment on: Meltzer AC, Burrows PK, Wolfson AB, et al. Effect of tamsulosin on passage of symptomatic ureteral stones: a randomized clinical trial. \\ JAMA Intern Med 2018;178:1051-7.
}

Submitted Aug 25, 2018. Accepted for publication Sep 07, 2018.

doi: 10.21037/atm.2018.09.09

View this article at: http://dx.doi.org/10.21037/atm.2018.09.09

Over ten years ago, the use of $\alpha$-blockers like tamsulosin as medical expulsive therapy (MET) evolved into a widely accepted and popular treatment for conservative management of ureteral stones. In the early beginnings, there were mainly small studies of various quality and results, but evidence for the effectiveness of the treatment was underlined by several systematic reviews and metaanalysis $(1,2)$. But even after inclusion of MET in the major guidelines as accepted treatment method, there was a need for further studies to raise the level of evidence (3). Recently, several different study groups published high-quality randomized controlled trials (RCT) with a sufficiently powered study population and rigorous methodology, coming to different conclusions, but in general questioning the broad use of MET especially for ureter stones $<5 \mathrm{~mm}$.

After publication of the first negative results in those high-quality RCTs, a discussion aroused about the cause of the divergence to the findings of meta-analysis published beforehand. New questions including the role of subgroup analyses formed, which ultimately lead to further investigations.

The most recent study about MET in 7AMA Internal Medicine: "Effect of Tamsulosin on Passage of Symptomatic Ureteral Stones, a Randomized Clinical Trial”, published by Andrew C. Meltzer and colleagues, concluded that stone passage rates did not differ significantly between the treatment and placebo groups. They randomized 512 patients to either tamsulosin or placebo for 28 days and assessed stone passage by visualization through computed tomography (CT) or physical capture of the stone. It was a two-staged approach with an initial open-labelled phase at a single-site to assess the feasibility of recruitment and to revise the original estimate of sample size for the trial. The second phase was performed in six emergency departments throughout the U.S. with a telephone-based follow-up at different time points until 90 days after randomization and a follow-up CT-scan 28 days after initial presentation and start of the treatment.

Besides a similar stone passage rate, secondary outcomes like crossover to open label tamsulosin, surgery for urinary stone, hospitalization, return to work or analgesic use did also not differ significantly between the two treatment arms. Besides the rate of ejaculatory dysfunction, the treatment related side effects or adverse events did not differ either.

As different studies found conflicting results for bigger stones $\geq 5 \mathrm{~mm}$ in the distal ureter, they also performed post hoc analysis including location in the ureter and stone size. They did not find a significant interaction with treatment. However, the study was not designed to detect treatment effect based on stone size and with a mean diameter of $3.8 \mathrm{~mm}$, a high proportion of the participants had stones $\leq 5 \mathrm{~mm}$ (4).

These results are consistent with the SUSPEND trial, published in The Lancet in 2015 by Pickard et al., a threegroup, randomized, placebo-controlled trial of medical expulsive therapy (tamsulosin $400 \mu \mathrm{g}$, nifedipine $30 \mathrm{mg}$ or placebo) in adults with ureteric colic. In this study 1,167 
patients got randomized in one of the three treatment arms with a primary outcome defined as the proportion of participants not needing any further intervention for stone clearance within four weeks of randomization. They reported no significant difference between active treatment and placebo group, or between tamsulosin and nifedipine. They therefore stated: $\alpha$-blockers are not effective in the treatment of ureter stones. But again, the average stone size in this patient population was $<5 \mathrm{~mm}(4.5 \mathrm{~mm})$ and with only one fourth of the stones $\geq 5 \mathrm{~mm}$ the subgroup analysis was not powered to evaluate the effectiveness in bigger stones (5).

This study initiated a discussion about the standardization of patient outcome reporting. "No need for further intervention for stone clearance" as the chosen outcome may be clinically relevant, but is not widely used and very heterogeneous due to varying treatment algorithms. It leads to speculations weather the results could have been different if the primary outcome of the study had been confirmed stone expulsion or absence of a stone on proper imaging, a precisely measurable and easier comparable variable.

A subsequent methodological thorough, randomized controlled multi-center study with an acceptable patient population of 403 patients was published half a year later by Furyk et al. The Australian research group concentrated on symptomatic distal ureter stones and prespecified subgroups of stones less than $5 \mathrm{~mm}$ and 5 to $10 \mathrm{~mm}$. The primary endpoints were stone expulsion on CT at 28 days and time to stone expulsion. They also found that treatment with tamsulosin did not affect stone passage overall. Also, time to stone passage, pain, or analgesia requirements did not differ significantly. In the subgroup analysis on the other hand, they could show a significant increase of spontaneous passage in the treatment group and therefore recommended tamsulosin for distal ureter stones $\geq 5 \mathrm{~mm}$ (6).

Considering these findings, a Chinese study group, led by Ye et al., conducted a double-blind, placebo-controlled study of 3,296 patients with distal ureteral stones, across 30 centers, to evaluate the efficacy and safety of tamsulosin. At present, it is the largest study focusing on large stones between 4 and $7 \mathrm{~mm}$ diameter in the distal ureter, the patient cohort most likely to benefit from MET according to previous studies. Concerning the methodological quality, the authors implemented concealed random allocation stratified by stone size, blinding of patients, personnel, and outcome assessors and an intention-to-treat analysis based on an a priori protocol to avoid any bias. For detection of the overall stone expulsion rate, Ye et al. had chosen weekly CT imaging over a 28-day surveillance period. This rigorous follow-up is unlikely to be repeated at other institutions due to stringent radiation safety protocols, like the ALARA (As Low as Reasonably Achievable) principle for example (7). However, ultimately they could prove significant effectiveness of tamsulosin compared to placebo for the treatment of distal ureteral stones $>5 \mathrm{~mm}(86 \%$ vs. $79 \% ; \mathrm{P}<0.001)$. They also found significant favoring outcomes for their secondary outcomes including the requirement of less analgesics $(\mathrm{P}<0.001)$, a lower incidence of renal colic for all stone sizes $(4-7 \mathrm{~mm})(\mathrm{P}<0.001)$ and shorter time to expulsion $(\mathrm{P}<0.001)$ in the tamsulosin group (8). But while this study will be influential in upcoming guideline discussions, there are inconsistencies still unacknowledged. The high rate of stone sizes between $6-7 \mathrm{~mm}(64 \%)$ in comparison to $4-5 \mathrm{~mm}(34 \%)$ was divergent to previous reported studies $(5,6)$ and could indicate a patient selection exceeding the already existing limitation of distal ureter stones $4-7 \mathrm{~mm}$ in diameter.

The latest results from the MIMIC study, a multi-center international cohort-study in 71 centers led by Shah et al. via the UK British Urology Researches in Surgical Training (BURST) have been presented at the EAU congress in Copenhagen in March 2018. They included data from 4,181 patients presenting with acute ureteric colic of whom $75 \%$ $(n=3,127)$ were discharged with conservative treatment. Thereof $80 \%(n=2,516)$ of the patients had a spontaneous stone passage and were included in a multivariable analysis including MET use, age, gender, stone size and stone position. They did show no significant association of MET use with spontaneous stone passage irrespective of stone size or location in the ureter (9). But as the results aren't published so far we still need to await final release to consider these findings in our daily practice.

While there came up several opposing recommendations in various randomized controlled trials, some of them mentioned above, the American, European and German guidelines still recommend MET for conservative management of distal ureter stones $5 \mathrm{~mm}$ or larger $(10-12)$. Based on the revision of the latest evidence, we agree that these guidelines should be unified supporting the usage of $\alpha$-blockers as medical expulsion therapy as one of the treatment options for ureteral stones $\geq 5 \mathrm{~mm}$. But as there may be beneficial effects on the analgetic use and time to expulsion, MET could also be offered to informed patients with smaller stones. Therefore, patients need to be properly educated about the benefits and risks before deciding for MET. 


\section{Acknowledgements}

None.

\section{Footnote}

Conflicts of Interest: The authors have no conflicts of interest to declare.

\section{References}

1. Hollingsworth JM, Rogers MA, Kaufman SR, et al. Medical therapy to facilitate urinary stone passage: a metaanalysis. Lancet 2006;368:1171-9.

2. Seitz C, Liatsikos E, Porpiglia F, et al. Medical therapy to facilitate the passage of stones: what is the evidence? Eur Urol 2009;56:455-71.

3. Campschroer T, Zhu Y, Duijvesz D, et al. Alpha-blockers as medical expulsive therapy for ureteral stones. Cochrane Database Syst Rev 2014;(4):CD008509.

4. Meltzer AC, Burrows PK, Wolfson AB, et al. Effect of Tamsulosin on Passage of Symptomatic Ureteral Stones: A Randomized Clinical Trial. JAMA Intern Med 2018;178:1051-7.

5. Pickard R, Starr K, MacLennan G et al. Medical expulsive therapy in adults with ureteric colic: a multicentre, randomised, placebo-controlled trial. Lancet 2015;386:341-9.

Cite this article as: Veser J, Seitz C. The status of medical expulsive therapy in the age of evidence-based medicine. Ann Transl Med 2018;6(Suppl 1):S18. doi: 10.21037/atm.2018.09.09 